\title{
Design of Variable-Friction Devices for Shoe-Floor Contact
}

\author{
Guillaume Millet ${ }^{\mathrm{a}}$, Martin Otis ${ }^{\mathrm{b}}$, Daniel Horodniczy ${ }^{\mathrm{a}}$, Jeremy R. Cooperstock ${ }^{\mathrm{a}, *}$ \\ ${ }^{a}$ McGill University, Centre for Intelligent Machines, 3480 Rue University, Montréal, Québec H3A OE9 \\ ${ }^{b}$ REPARTI Centre, Université du Québec à Chicoutimi, 555 Boulevard de l'Université, Chicoutimi, Québec G7H $2 B 1$
}

\begin{abstract}
In rehabilitation training, high-fidelity simulation environments are needed for reproducing the effects of slippery surfaces, in which potential balance failure conditions can be reproduced on demand. Motivated by these requirements, this article considers the design of variable-friction devices for use in the context of human walking on surfaces in which the coefficient of friction can be controlled dynamically. Various designs are described, aiming at rendering low-friction shoe-floor contact, associated with slippery surfaces such as ice, as well as higher-friction values more typical of surfaces such as pebbles, sand, or snow. These designs include an array of omnidirectional rolling elements, a combination of lowand high-friction coverings whose contact pressure distribution is controlled, and modulation of low-frequency vibration normal to the surface. Our experimentation investigated the static coefficient of friction attainable with each of these designs. Rolling elements were found to be the most slippery, providing a coefficient of friction as low as 0.03 , but with significant drawbacks from the perspective of our design objectives. A controlled pressure distribution of lowand high-friction coverings allowed for a minimum coefficient of friction of 0.06 . The effects of vibration amplitude and frequency on sliding velocity were also explored. Increases in amplitude resulted in higher velocities, but vibration frequencies greater than $25 \mathrm{~Hz}$ reduced sliding velocities. To meet our design objectives, a novel approach involving a friction-variation mechanism, embedded in a shoe sole, is proposed.
\end{abstract}

Keywords: variable friction, foot-floor interaction, rehabilitation, balance training

\section{Introduction}

Friction is usually quantified to a first approximation with Coulomb's model, in which a relative motion between two solids starts when the ratio of tangential (frictional)

5 to normal forces exceeds a certain value, known as the coefficient of static friction $\left(\mu_{\mathrm{s}}\right)$. In the context of walking, friction is the force resisting the relative motion between the floor surface and a walker's shoe.

Recent work on tactile feedback in human-computer interaction (HCI) has recognized the importance of modulation of friction as a complement to the perceptual effects induced by vibration alone. Popular examples include applications to smartphones and tablet screens to improve performance and emotional response with touch interactions, by using ultrasonic vibrations [1, 2] or electrovibration [3], but also to everyday objects [3]. However, despite considerable exploration of VR gaming interfaces intended for walking and running, ${ }^{1}$ as well as smallsize [4, 5] and large-size [6] computer-controlled, omni-

\footnotetext{
* Corresponding author

Email addresses: guillaume@cim.mcgill.ca (Guillaume Millet), Martin_0tis@uqac.ca (Martin Otis), danielh@cim.mcgill.ca (Daniel Horodniczy), jer@cim.mcgill.ca (Jeremy R. Cooperstock)

${ }^{1}$ http: //www.virtuix.com
}

20 force feedback [7], rotary treadmill [8], and stepping platforms $[9,10]$, there has been little attention to achieving similar variable-friction capability for foot-based interaction. Variable ground-surface friction could have important applications for HCI, for example, hands-free interaction with virtual sliders [11], entertainment, such as amusement park rides, games, simulation training, and rehabilitation.

The research described in this manuscript is motivated by the problem of risk of falls, in particular among elderly and post-stroke populations, arising from foot-ground contact with unexpectedly slippery surfaces. One approach adopted by some rehabilitation centres employs artificial ice rinks (in miniature) to reproduce the conditions of icy ground, allowing for subjects to gain familiarity with such conditions in a safely controlled environment. However, these do not allow for the experience of unexpected slippery conditions, as results, for example, when encountering a patch of ice on the sidewalk. Our long-term objecheterogeneous real-world ground environments, including the variability of coefficients of friction that we experience in everyday walking activities, so as to improve the quality of training and rehabilitation that can be offered.

As an initial step toward this objective, we sought to 
characterize the tradeoffs between various design options, beginning with the challenge of achieving a minimal coefficient of friction approximating that of ice, and considering the possibilities for varying the coefficient of friction dynamically, on demand. This exploration of the design space is necessary to select suitable avenues for further development of functional prototypes, and ultimately, to test these prototypes with human subjects.

The contributions of the present work include the implementation of several prototypes for a variable-friction dis ${ }_{110}$ play as well as their assessment, motivated by the demands of rehabilitation applications, in which high-fidelity vibrotactile and acoustic rendering through the floor interface are critical to the intended perceptual effects. At a high level, this involves two basic design strategies, one using rolling elements and another with low-friction coverings. Since the latter approach is more suitable for our target applications, mechanisms for controlling the contact pressure distribution between pairs of different materials are investigated further. Finally, we consider the possibility of $\mathbf{1}_{\mathbf{2}}$ realizing this variable-friction function through the implementation of an in-sole mechanism, which offers significant advantages of reduced size and cost of implementation. Integration of vibrotactile rendering capability, related to the simulated ground surface, in conjunction with the controh ${ }_{25}$ of variable friction under the foot, remains a challenge for future investigation.

\section{Background}

\subsection{Balance Failures}

75 Unintentional falls are a leading cause of serious injury, loss of independence, and even death, especially among the elderly. Statistics from the United States [12], Canada [13], China [14] and Finland [15] indicate that one third of ${ }_{135}$ adults aged 65 and over will fall at least once every year, causing up to $88 \%$ of injuries in this age group [13]. Considering the numerous problems that a fall can cause, many research programs have developed gait analysis systems. Similarly, human-centered approaches have been used to estimate slipping and falling hazards along with the asso-140 ciated risks [16].

The etiology of slip and fall accidents was surveyed by Gao and Abeysekera [17] who provided a systematic analysis of the various contributing factors and described means of prevention, in particular for icy and snowy surfaces $\mathbf{1 4 5}$ One preventive approach used to reduce the frequency and severity of falls is to change human behavior with the aid of a balance-training program, which uses different tools for improving balance control over different surfaces [18, 19]. Real outdoor trails are often used, with a wide range of ob-150 stacles that may be encountered in everyday life, consisting of different sections covered with rocks, sand, pebbles or gravel. However, the diversity of soil types available for evaluation or training may be limited, and seasonal conditions in northern regions do not allow for year-round uses5 of such facilities.

\subsection{Virtual Environments for Balance Training}

An alternative is the use of simulated environments, for example, as employed in rehabilitation institutes, to train balance and mobility functions. These may reproduce specific environmental conditions with high ecological validity [20, 21]. Similarly, home-based systems have emerged, including those based on the Nintendo Wii Fit [22, 23], although the degree to which balance-failure conditions can be manipulated, while presented naturally, is generally limited. Such unsupervised systems are not intended to simulate different levels of friction, nor would it be safe to do so outside of a clinical setting where training can be supervised.

Virtual reality rehabilitation protocols may significantly improve the quality of treatment by offering strong functional motivations to the patient, who becomes more attentive to the movement to be performed [24, 25]. Such training in the post-stroke population has demonstrated encouraging results for improving gait speed, endurance, and force production [21, 26]. Moreover, Bhatt and Pai demonstrated that the locomotor-balance skills acquired with the aid of low-friction movable platforms could translate into greater ability to avoid falls encountered in daily living [27].

Reproducing the unexpected nature of real-life slipping accidents is one of the challenges of using a simulation system to induce imbalances during walking [28]. Whereas natural ground surfaces may exhibit significant variation in the coefficient of friction, most simulation systems proposed to date provide either a single coefficient of friction, or, for the case of setups employing "lockable" passive rolling elements, at most two levels of friction $[29,30]$. Moreover, these systems are unable to convey the tactile properties of ground surfaces, which may play a role in balance perturbations. Other systems employ active moving surfaces to induce imbalances [31] but these do so without controlling friction.

\subsection{Effects of floor slipperiness on locomotion}

Human-centered studies of balance, gait biomechanics, and motor patterns in neurophysiology have provided an improved understanding of various human behaviors associated with falls and slips.

Overall, subjects confronted with a slippery floor increase limb stiffness and adopt a gait that tends to maintain the center of mass centered over the supporting limbs. In their study, Cham and Redfern [28] found that healthy humans change their gait when there is a potential risk of slipping, even when asked to walk as naturally as possible. The gait changes included reductions in stance duration and loading speed on the supporting foot, shorter normalized stride length, reduced foot-ramp angle and slower angular foot velocity at heel contact. These postural and temporal gait adaptations reduced the potential of slips and falls by $16-33 \%$ on average. Greater toe grip and gentler heel strike [32] or greater knee flexion torque [33] were also observed as adaptation strategies to slippery floors . 
Cappellini et al. [34], who combined measurements of gait kinematics, ground reaction forces, and bilateral electromyographic activity, reported similar features of walkng on a slippery surface. These included smaller step length, cycle duration, and horizontal shear forces, in ad-215 dition to stable head orientation. They also reported increased arm movements, trunk rotations, and lateral trunk inclinations, and noted foot motion and gait kinematics hat avoided the entire sole in contact with the ground. Using similar measurement techniques, Oates et al. [35 220 showed that knowledge of and experience with a slippery surface result in proactive and reactive adjustments in behavior to stop on a slippery surface more effectively and safely.

The experimental devices used in those slips-related25 studies to simulate slippery floors or to engender a slip were based on different methods of friction reduction. The following section presents those methods among other potential ones.

\subsection{Variable-Friction Walking Devices}

The coefficients of static friction encountered in typical walking scenarios can vary widely. For instance, rubber on dry asphalt has a $\mu_{\mathrm{s}}$ above 0.5 and rubber on ice can drop $\mu_{\mathrm{s}}$ as low as 0.02 when temperature is near $0^{\circ} \mathrm{C}[36]^{35}$ or when only a thin layer of snow is present on the contact area [37]. Depending on the application, providing such a low $\mu_{\mathrm{s}}$ may be the goal, for instance when studying slipinduced falls. In other contexts, such as entertainment ations, this may be a problematic safety iss thus, not recommended. The required friction to walk on a dry, level surface without slipping was reported by various studies to lie between $0.17-0.23$ [38].

Designing a floor or shoe device able to generate such a ide range of friction coefficients when people walk on ores with it poses two interdependent issues: how to achieve, using little energy, very low friction approaching $\mu_{\mathrm{s}}$ of 0.02 and how to be able to vary this friction both continually and controllably?

In mechanical systems design, reducing friction between ${ }^{50}$ surfaces is usually accomplished by one of the three following methods:

- Lubrication: changing dry friction into lubricated friction by placing a lubricant such as oil, water, or grease between the two surfaces.

- Materials: using naturally low-friction materials such as ultra-high-molecular-weight polyethylene (UHMWPE) commonly found in synthetic ice rinks, or polytetrafluoroethylene (PTFE), whose self ${ }^{\mathbf{2 6 0}}$ contact $\mu_{\mathrm{s}}$ ranges from 0.05 to 0.1 at low sliding speeds $(<0.1 \mathrm{~m} / \mathrm{s})$ [39].

- Rolling: changing sliding friction into rolling friction, which naturally has a lower resistance. Components such as rolling-element bearings often allow $\mu_{\mathrm{s}}$ below 0.02 .
Experimental devices used in slip-related studies have usually been based on the three methods mentioned above. Most varied the slipperiness by changing the materials and the lubrication, in other words, the floor-footwearcontaminant conditions [40, 41, 42, 28, 33, 32, 38, 43, 34]: subjects walked along a walkway with different flooring material, e.g., vinyl, PTFE, carpet, plywood, or concrete, over which a contaminant such as water, sand, soap, diluted glycerol, or oil could be poured, and in different shoe conditions, e.g., different sole material, sole hardness, and heel height. The lubrication approach offers advantages of very low friction but is not suitable for our design goals because of the difficulty in removing the lubricant quickly and varying the friction in a controllable manner. Although not considered here, the reader interested in investigating the use of lubrication to vary friction would likely benefit from the experimental analysis and modelling work on lubricated shoe-floor friction by Beschorner et al. $[44,45,46]$.

Other researchers have used a roller-based apparatus $[29,47,35]$, which can exhibit two levels of friction (nonslippery when the rollers are locked and very slippery when unlocked). ${ }^{2}$ However, this adds significant perceptual biases such as an uneven surface, vibrotactile noise, visibility (awareness of the danger) and friction anisotropy. A mechanism to modulate the available friction on such a roller-based system, continuously, was proposed previously by Millet et al. [48]. Pai et al. have used a similar approach with a mobile platform atop a set of lockable low-friction linear bearings [30, 49, 27], which avoids some of the aforementioned drawbacks such as surface irregularity and visibility, but introduces another behavioral bias due to platform inertia and requires a platform as long as the sliding length, which limits its applicability.

Air bearings offer a method similar to lubrication by utilizing a thin film of pressurized air between surfaces, but this also seems impractical for a walking device. If the pressurized air supply were connected to each shoe, as an air caster, this would impede free movement of the legs during walking. Alternatively, if it were connected to the floor, as an air hockey table, it would be challenging to supply sufficient pressure under the shoe at arbitrary orientations, particularly during the initial contact and pushoff phases when the foot is inclined.

Ultrasonic vibrations ( $>20 \mathrm{kHz}$ ) have been used recently to create variable-friction tactile displays capable of rendering virtual textures under the fingertip. The reduction of friction is caused by the creation of a squeeze film of air between the vibrating surface and the finger touching it [50]. This piezo-actuated technology was first implemented in variable-friction touchpads [51, 52], and later as variable-friction touchscreens $[1,53]$, with the latest developments aimed at improving the resolution and flatness of the frequency response with which the effects

${ }^{2}$ This approach was commercially popularized by roller shoes http://www.heelys.com. 

forces and sizes of feet are at least two orders of magnitude higher than those of fingers, this technique of friction reduction is not likely to scale to floor applications.

Another method to reduce friction, although uncomincrease repulsive forces between contact surfaces, particularly with electromagnetism. Such a system would require control of an array of coils embedded in the floor, which would apply a repulsive force to magnets embedded in the shoes. In this way, Berkelman to control the orientation and the position of a magnet throughout a large workspace. However, the scalability to human walking seems improbable, as it would require an unsafe, heavy magnet in the shoe and a very complex control system.

Finally, some work has been done to adjust the sliding characteristics of the shoe, in particular for experienced ${ }^{32}$ bowlers who desire different sliding friction on the floor next to the bowling lane. For instance, Lewia designed a shoe heel with a reversible asymmetric profile that exhibits two different levels of friction depending on the user's heel orientation [58]. Similarly, Pasternak allowed for the user ${ }^{325}$ to obtain continuous variation in friction by tilting the heel or foresole portions of the sole such that the contact area is changed. However, the variation is controlled by the user manually, and attains only non-slippery levels of friction. Another related design includes a swivel inside the sole ${ }^{330}$ that allows the user to spin about a vertical axis [59] but does not support variable friction or slipping movements.

\section{Design strategies for variable friction}

Our target applications require variation in shoe-floor friction ranging from non-slippery to slippery levels. At the latter end, the importance of achieving potentially very slippery effects encouraged us to test the use of rolling el ${ }_{340}$ ments initially. This section presents the design requirements of such an approach compared to alternatives employing thin surface coverings.

\subsection{Rolling Elements}

Rolling elements have been used recently in a twoenal, treadmill-like locomotion interface using an array of balls that are actuated from underneath by a belt mounted on a turntable [8]. All of the rolling elements, however, are driven by a single belt, and therefore, cannot induce relative motion between the feet. Actuating all thes5o elements individually would be prohibitively expensive and would require precise measurements of normal and tangential forces to simulate variable friction correctly.

Since the device intended here is not a locomotion interface, another solution is to let the balls roll freely under

315 the shoes and provide a mechanism to vary the resistance of motion. Such free omnidirectional rolling capability is provided by ball transfer units (BTUs), whose principle

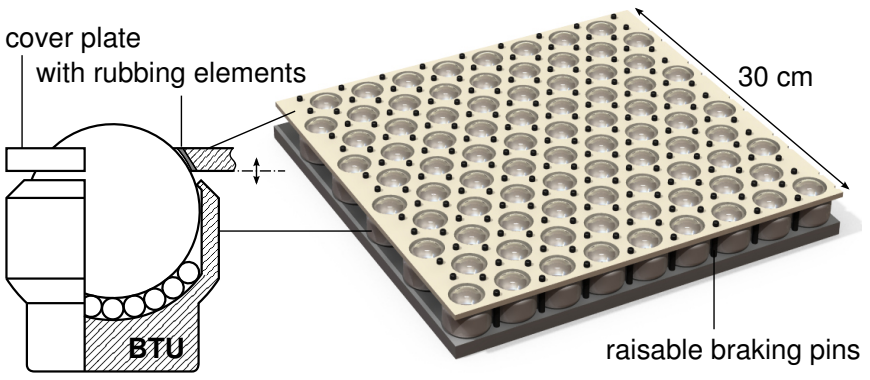

Figure 1: CAD view of a conceptual 2D low-friction floor tile made of ball transfer units. Friction could be varied either by pressing a cover plate against the balls or by raising braking pins between the units.

of operation is identical to that of a trackball. As illustrated in Fig. 1, a BTU involves a large load-bearing ball supported by many smaller balls encapsulated in a hemispherical cup. These are commonly used in an array to move objects with minimum effort in any direction, e.g., in cargo and baggage handling applications. The need for small balls under the load-bearing ball precludes the design of a braking mechanism in contact with the ball from underneath.

Achieving rolling friction over a flat surface requires packing the array units very closely together, made possible by the use of small diameter balls and minimal surrounding hardware. Bolt or glide units $^{3}$ are simple and inexpensive and their large ball exposure can be advantageous in the design of a braking mechanism for varying the friction. Commercial glide units contain a $\varnothing 25 \mathrm{~mm}$ ball, allowing for construction of an array of 85 units on a $30 \times 30 \mathrm{~cm}$ tile (see Fig. 1). In order to avoid jamming at heel strike, a low-friction cover plate above the BTUs' frames would be used to flatten the surface, so that the balls protrude only slightly, e.g., in the order of a few millimeters, out of the plate.

Friction variation of a BTU-based floor tile could be accomplished in different ways. The rolling friction of the balls could be varied by controllably pressing a rubbing element against each ball on its exposed part with the aforementioned cover plate (see Fig. 1). Another way is to provide an array of raisable braking pins into the surface of the floor between the BTUs such that, when raised, they rub against the shoe and increase friction. Different levels of friction could thus be obtained based on the number, the size, the height, and/or the material of the raised pins.

\subsection{Low-Friction Surface Coverings}

A design employing a rubbing mechanism in conjunction with low-friction materials instead of BTUs could also be used to achieve variation of friction. This alternative requires covering both the shoes and the tile with

\footnotetext{
${ }^{3} \mathrm{SFK}$ publication 940-711, http://www.skf.com/binary/ 30-285023/Ball-Transfer-units .pdf
} 


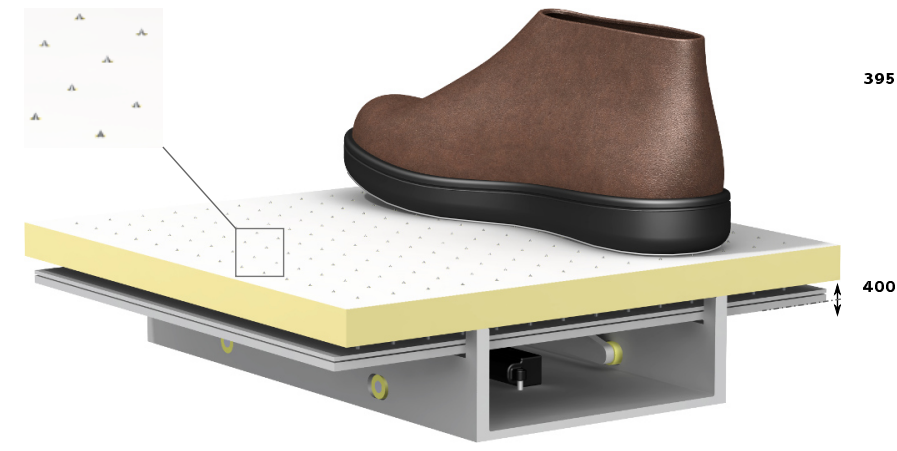

Figure 2: CAD view of $2 \mathrm{D}$ variable-friction floor tile using low-405 friction covering and sharp pins. The sole of the shoe is also covered with a low-friction film. The coefficient of friction would be controlled by varying the protuberance of the sharp pins.

the materials, for example PTFE or UHMWPE, whose coefficient of friction defines the lowest friction achievable by ${ }^{\mathbf{4 1 0}}$ the device. The materials should be wear resistant during walking. Although PTFE is theoretically more slippery than UHMWPE, it is softer and therefore more prone to indentations from dust particles crushed under the shoe. Apart from one study using PTFE flooring ${ }^{415}$ with participants wearing only socks [40], slip-related experiments have usually preferred the use of vinyl or wood covered with oil or soap, instead of PTFE, for the slippery condition. Our initial tests investigated PTFE and UHMWPE coverings, but the choice of optimal material ${ }^{420}$ for this application remains an open question.

For two surfaces in sliding contact, friction variation requires a modification of one or both of the surfaces, which, in our case, may be either the tile surface or the sole surface. This can be realized by a change in the texture using ${ }^{425}$ a rubbing mechanism. The size of the rubbing contact translates approximately to two classes of solution: sharp pins modify the macroscopic texture, similar to epoxy non375 slip coatings, whereas large sliding contact areas affect the microscopic texture, resulting in a perceived change of ma- ${ }^{430}$ terial.

\subsubsection{Variable-friction device with sharp pins}

Figure 2 illustrates the conceptual design of a floor tile $e_{435}$ employing this technique. Importantly, this design is suitable for use as a video projection surface. The tile consists of a $30 \times 30 \mathrm{~cm}$ plate covered with a low-friction material through which an array of sharp pins can slide to vary the coefficient of friction according to their protuberance. The actuation force on the array of pins would be applied symmetrically through a cross mechanism to avoid butting of the pins. The frame can be mounted on load cells to measure foot-applied forces for the purpose of biomechanical analyses or for closed-loop control of the friction.

An important design parameter in using sharp pins is their spatial resolution, as this affects the effective friction for small contact areas, such as at heel strike when the contact area can be as small as $1 \mathrm{~cm}^{2}$. The array of pins therefore needs to be sufficiently dense, while remaining in the limit of the mechanical feasibility and strength of the surface. For example, a $1 \mathrm{~cm}$ spacing in a $30 \times 30 \mathrm{~cm}$ plate induces an array of 900 pins, which implies a significant cost of machining and assembly. Also, the size of the tiles over which friction must be controlled impacts on the complexity and the achievable spatial granularity at which friction may be controlled. With smaller tiles, the actuation mechanism could be simpler but more electronics would be required to control the network of tiles. These drawbacks impede the construction of large variable-friction floors.

Instead, the variable-friction mechanism could be built into the shoe sole itself, leaving the floor covered only with a low-friction material. Regardless of whether they are built into the floor or the shoe, however, using sharp pins has the disadvantage of wearing down the coverings.

\subsubsection{Variable-friction shoe with soft rubbing areas}

Certain foam rubbers can present high friction when pressed against PTFE or UHMWPE. For example, we measured values of $\mu_{\mathrm{s}}$ of $0.55-0.65$ for gum foam rubber and 0.9-1.1 for neoprene rubber, both against UHMWPE. Therefore, the effective friction can be varied by combining such low- and high-friction materials, and controlling the normal force applied to the two rubbing materials.

This principle of friction variation is illustrated in Fig. 3. To allow a continuous variation, the normal force applied by one of the two rubbing materials needs to be adjusted relative to the other. When these are pressed against a flat floor, the normal forces that are applied depend on the relative offset and stiffness of the structure between the rubbing materials. For a solid structure, only microscopic offsets can be achieved, which would be difficult to control. To allow for a wider range of offsets, an elastic element is inserted between the shoe and the controlled rubbing surface. This permits greater variation in the distribution of applied forces to the rubbing materials, a corresponding wider range of motion of the compressing mechanism, and in turn, an increased ability to vary the friction. For our application, we decided to control the position of the highfriction material, so that when the elastic element is not stretched, the effective available friction is that of the lowfriction pair of materials and the contact is slippery.

Assuming Coulomb's model and that the mass of the device is negligible compared to the weight of the user, the effective coefficient of friction, $\mu_{\text {eff }}$, can be derived easily as

$$
\begin{aligned}
F_{\text {human }} & =F_{\mathrm{lf}}+F_{\mathrm{hf}} \\
F_{\mathrm{hf}} & =E_{\mathrm{el}} S_{\mathrm{el}} \varepsilon_{\mathrm{el}} \\
\mu_{\mathrm{eff}} & =\frac{\mu_{\mathrm{lf}} F_{\mathrm{lf}}+\mu_{\mathrm{hf}} F_{\mathrm{hf}}}{F_{\mathrm{lf}}+F_{\mathrm{hf}}} \\
\mu_{\mathrm{eff}} & =\mu_{\mathrm{lf}}+\left(\mu_{\mathrm{hf}}-\mu_{\mathrm{lf}}\right) \frac{E_{\mathrm{el}} S_{\mathrm{el}} \varepsilon_{\mathrm{el}}}{F_{\text {human }}}
\end{aligned}
$$

where $F_{\text {human }}$ is the vertical force applied by the user on the shoe (weight and inertial force of the user), $F_{\text {lf }}$ and 


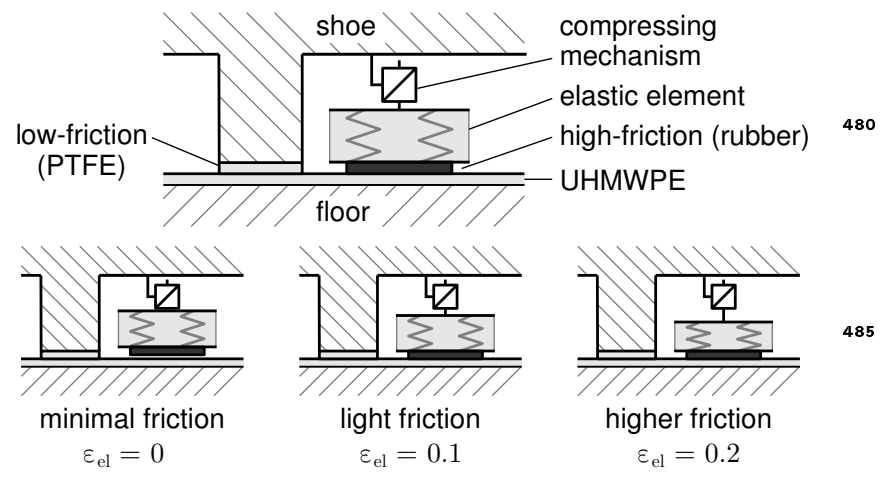

Figure 3: Principle of the friction variation with two different pairs of materials. The strain of the elastic element $\varepsilon_{\text {el }}$ changes the force equilibrium between the low- and high-friction surfaces, thereby varying ${ }_{490}$ the effective coefficient of friction.

$F_{\text {hf }}$ are the normal forces applied on the low-friction and high-friction surfaces, respectively, $E_{\text {el }}$ is the Young's modulus of the elastic element, $\varepsilon_{\mathrm{el}}$ its controlled strain, $S_{\mathrm{el}}$ its $^{49}$ cross-sectional area, and $\mu_{\mathrm{lf}}$ and $\mu_{\mathrm{hf}}$ are the coefficients of friction of the low-friction and high-friction surface, respectively. When the elastic elements are at rest, i.e., $\varepsilon_{\mathrm{el}}=0$, then $\mu_{\text {eff }}=\mu_{\mathrm{lf}}$. The compression of the elastic element

445 increases the force applied to the high-friction surface $F_{\mathrm{hf}}{ }^{50}$ by an amount equal to the decrease of force applied to the low-friction surface $F_{\mathrm{lf}}$. If $F_{\mathrm{hf}}$ reaches the total force $F_{\text {human }}$, then the low-friction surface does not touch the floor and $\mu_{\mathrm{eff}}=\mu_{\mathrm{hf}}$.

Although the principle seems simple, its application to $\mathrm{a}^{\mathbf{5 0 5}}$ walking shoe raises some challenges because $F_{\text {human }}$ varies during a stride. Moreover, if the elastic element is distributed over the high-friction surface at several different locations under the shoe, then $S_{\text {el }}$ will also vary during a stride. Indeed, the high-friction surface has to be present ${ }^{510}$ in the back of the sole for the heel strike phase, in the front for the toe-off phase, and in at least one place in between for the transition. Therefore, in order to control the effective friction regardless of foot orientation, $S_{\text {el }}$ and $F_{\text {human }}$ must be estimated and used as parameters of a real-time controller that adjusts the strain of the elastic element. Both estimations can be obtained by the use of thin force $\mathrm{F}_{\mathbf{5 1 5}}$ sensors such as force-sensing resistors, whose thickness is less than $0.5 \mathrm{~mm}$.

The pairs of rubbing materials used define the theoretical range of effective friction achievable. For the lowfriction condition, a pairing of PTFE and UHMWPE masıo terials seems to be preferable to PTFE with PTFE because the latter combination exhibited a noticeable difference between static and dynamic friction in our initial tests. A similar comparison led us to choose a pairing of rubber and UHMWPE for the high-friction condition. UHMWPE is preferable for the floor covering owing to its superior wear resistance.

From (4), the other factor contributing to the effective friction is the elastic element. The maximal weight applied and the maximal desired friction give a value for $E_{\mathrm{el}} S_{\mathrm{el}} \varepsilon_{\mathrm{el} \text {,530 }}$ where the strain $\varepsilon_{\mathrm{el}}$ is the ratio of total deformation to uncompressed length of the elastic element. The maximal compressed length results from a trade-off. On the one hand, it represents the maximal offset of the high-friction surface relative to the PTFE before the shoe touches the floor, since a large offset can impede the foot motion if it collides with the floor during the swing phase of walking. On the other hand, a small maximal offset makes control more difficult as it requires finer positioning. In the latter case, the effective friction will be more sensitive to mechanical tolerances and floor bumps.

\subsection{Adding Vibration to a Low-Friction Contact Surface}

Early work related to the friction and dynamics of machinery showed decreases of $90 \%$ in the static friction forces between steel samples with vibration frequencies above $40 \mathrm{~Hz}$. This provided evidence to explain the loosening of bolted joints in machines by the unscrewing of screws and nuts due to vibrations acting on the joints [60]. Further increases in either frequency or amplitude of vibration was found to result in decreased friction. Experiments on PTFE samples demonstrated reduction of friction by $35 \%$ for vibration amplitude of $200 \mu \mathrm{m}$ at $150 \mathrm{~Hz}$ [61]. Research has continued on the influence of oscillation on reducing static and sliding friction, particularly for ultrasonic vibrations [62], as well as theoretical modeling of that influence at the atomic scale [63].

The contact dynamics between the shoes and the floor vary with the compliance of the shoe and lower limb, in a way that certain frequencies and amplitudes of floor vibration can significantly decrease the normal forces at the contact, and in turn, result in lower friction forces. The resonance of the shoe creates a periodic oscillation of the normal force applied to the floor such that the static friction force oscillates and may become inferior to the external tangential forces, in which case, sliding may occur.

\section{Experimental evaluation}

Experimental exploration of the design space for variable-friction walking mechanisms focussed on the baseline characterization of methods that offered an ideal trade off between cost, controllability, ability to achieve low friction coefficients, and potential for large-scale implementation. As such, our evaluations were done on the following methods: rolling elements, surface coverings, and vibration addition. In each experimental subsection, a $\varnothing 100 \mathrm{~mm}$ steel cylinder, weighing $7.5 \mathrm{~kg}$, was used to ensure reliability of the measurements, since we found that we could not obtain acceptable standards of experimental consistency when pilot testing with human subjects. This eliminated a number of uncontrolled variables such as mass, stance and balance skill.

Our prototype slippery tiles are shown in Fig. 4. Since the tiles are intended to be part of a vibrotactile display, both the BTU-based and PTFE prototypes were built 

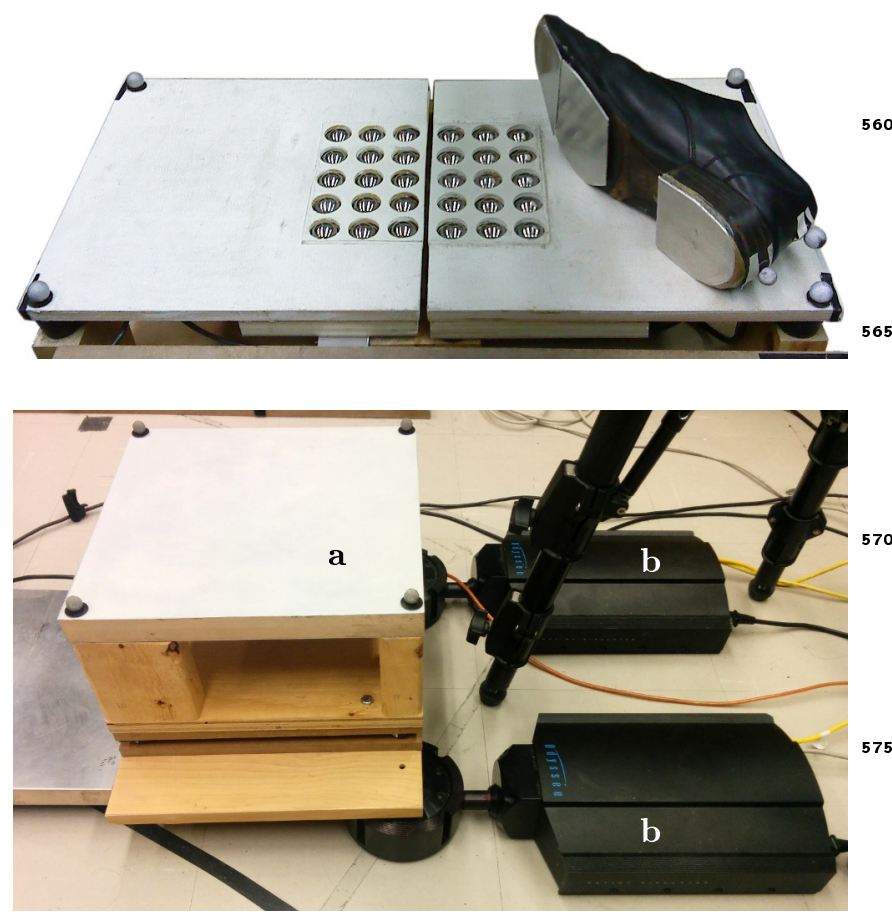

Figure 4: Prototypes of slippery tiles, using either BTUs (top) or a PTFE sheet (bottom), based on the vibrotactile tiles described irs80 reference [64]. The prototypes were fixed on a tilting mechanism in order to measure friction angles and investigate effects of vibration addition.

upon the vibrotactile tiles described in our previous work585 [64]. The elastomer elements originally fastened to the bottoms of the corners of the PTFE tile were removed to eliminate the damping effect they introduce to the setup.

The tiles were mounted on two different apparatuses, one of which was used to measure coefficients of static59o friction and the other to investigate the effects of vibration addition. The latter is shown in the bottom of Fig. 4. Static friction $\mu_{\mathrm{s}}$ was calculated from empirical measurements of the friction angle $\varphi_{\mathrm{s}}$ at which the shoe started sliding, as $\mu_{\mathrm{s}}=\tan \varphi_{\mathrm{s}}$.

Referring to the labels in the bottom image of Fig. 4, the tile a was raised from the right side by the linear actuators b (D-Box Odyssey, cutoff frequency: $100 \mathrm{~Hz}$ ), which also applied vibrations. The mechanism used to measure static friction angles consisted of a single D-Box actuatorøo that was able to tilt the prototype up to $10^{\circ}$. Tilting was performed with a slow ramp at a speed of $0.2^{\circ} / \mathrm{s}$. The orientation of the tile and the movement of the shoe were measured every $10 \mathrm{~ms}$ with a motion capture system consisting of three OptiTrack V100:R2 cameras.

\subsection{Rolling elements}

Experimental measurement of static friction of the BTU-based tile was carried out using a $0.6 \mathrm{~kg}$ hard soled men's dress shoe, shown in Fig. 4, and the $7.5 \mathrm{~kg}$ steel cylinder, shown in Fig. 5. The shoe had aluminium plates fastened to the bottom of the sole to avoid deformation due to contact pressure with the BTUs, which could result in uneven friction or in making contact with the tile. The shoe was placed at the center of the tile for each trial.

The friction angle was estimated at the beginning of sliding, associated with a change of velocity above $10 \mathrm{~mm} / \mathrm{s}$. After each test, the shoe was returned to its initial position for the following trial.

The results indicate a coefficient of $0.07 \pm 0.01\left(\varphi_{\mathrm{s}} \approx 4^{\circ}\right)$ for the BTU-based tile. Similar experimentation performed with human subjects, wearing shoes with the same aluminum plates covering the soles, indicates that coefficients as low as 0.03 are achievable using BTUs. BTUs are thus an attractive design option for devices intended to offer very low coefficients of friction. This is otherwise difficult to attain without the use of lubrication, which is impractical for controlled friction varying applications, as described in Section 2.4. Although the BTUs provide a low coefficient of static friction, able to simulate surfaces as slippery as ice, they suffer from some important limitations, as discussed in Section 5.1.

\subsection{Surface coverings}

A similar experiment was carried out to measure static friction of the PTFE tile, using the same shoe and mass, but covering the shoe sole with a PTFE sheet. To reduce the effect of dust, which significantly increased the resistance of motion, the PTFE surfaces were regularly wiped clean. The results indicate a coefficient of $0.11 \pm 0.01$ $\left(\varphi_{\mathrm{s}} \approx 6.3^{\circ}\right)$ for the PTFE tile as compared to $0.07 \pm 0.01$ for the BTU-based tile, described above.

Further experiments were conducted to evaluate the friction variation that could be obtained with a variablefriction shoe such as described in Section 3.2.2. To investigate the design factors related to the cross-sectional area, $S_{\text {el }}$, and the maximal offset of the elastic element, we used a test unit consisting of the same $7.5 \mathrm{~kg}$ steel cylinder, laid on a plate with a single high-friction surface, the rubber sheet shown on the right of Figure 5. The test unit was used to ensure adherence to our experimental reliability requirements. The PTFE surface was a $0.5 \mathrm{~mm}$ thick film made of Teflon ${ }^{\circledR} .4$ Its area was calculated such that the pressure applied on it was at least $0.34 \mathrm{MPa}$ in order to obtain a low coefficient of friction around 0.05 [39]. The rubber surface was a quick-recovery, $3 \mathrm{~mm}$ thick, soft sponge rubber sheet ${ }^{5}$ made of natural rubber, also called natural gum foam. ${ }^{6}$ The elastic element was a $13 \mathrm{~mm}$ thick EVA (ethylene vinyl acetate) foam pad. Its compression was adjusted by the number of paper sheets inserted between the wood base and the elastic element.

The static friction of the test unit was measured for different rubber offset positions, ranging from no contact to $2.2 \mathrm{~mm}$ (i.e., a strain of 0.14 ). Using similar measurement

\footnotetext{
${ }^{4}$ https://www.mcmaster.com/\#8569k45, Rockwell R60

${ }^{5}$ http://www.griswoldcorp.com/market/industrial.html, model 3110

${ }^{6}$ https : //wWw.mcmaster.com/\#8601k41
} 

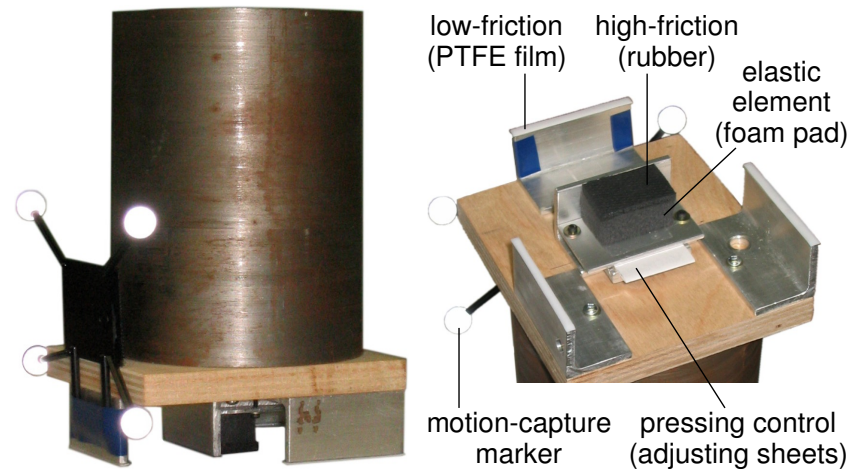

Figure 5: Test unit with $7.5 \mathrm{~kg}$ mass and variable-friction surface.

equipment to that described above, the test unit was laid on top of the tilting plate at 10 different locations to ensure uniform wear of the plate covering, which consisted of a $0.25 \mathrm{~mm}$ thick UHMWPE sheet. ${ }^{7}$ A second test was carried out with a rubber surface having twice the crosssectional area. The results for both tests are plotted in

$6 \mathbf{1 5}$ Fig. 6 and show good agreement with (4). In both tests, the effective friction increased linearly with the rubber deformation.

For the initial test, considering that the stiffness of the foam and rubber element was measured to be $4.7 \mathrm{~N} / \mathrm{mm}^{6}$ and $\mu_{\mathrm{hf}}-\mu_{\mathrm{lf}}$ to be 0.5 , (4) gives a rate of $0.031 \mathrm{~mm}^{-1}$ which is close to the observation of $0.028 \mathrm{~mm}^{-1}$ (error $<10 \%$ ). For the test with the larger rubber surface area, the measured rate was $0.057 \mathrm{~mm}^{-1}$, agreeing with the prediction of double the effective friction. The variability in the measurements was likely due to different states of wear of the plate covering.

In terms of load, the aforementioned strain of 0.14 corresponds to applying, out of the total load of $75 \mathrm{~N}, 10 \mathrm{~N}$ on the rubber surface and $65 \mathrm{~N}$ on the PTFE surface. In other words, shifting $13 \%$ of the total load to the rubber surface increased the static coefficient of friction from approximately 0.06 to 0.11 .

\subsection{Vibration addition}

The linear actuators described in Section 4 enable application of normal low-frequency vibrations up to $100 \mathrm{~Hz}$, by means of an audio signal to the controller (model KAI-1P). Acceleration of the PTFE tile was computed at the center of pressure of the force applied to the tile by the shoe. Details concerning the experimental measurement are provided in the Appendix. Force sensing was performed via four load cells (Measurement Specialties model FX1901) located under each corner of the support of the tile. Analog data from the force sensors were digitized via an acquisition board (National Instruments model USB-6218).

645 Experimental evaluation of the effect of vibration amplitude and frequency was carried out with the same $7.5 \mathrm{~kg}$ steel cylinder used in the preceding experiments.

\footnotetext{
${ }^{7}$ https ://www.mcmaster.com/\#85655k13, Durometer $70 \mathrm{D}$
}

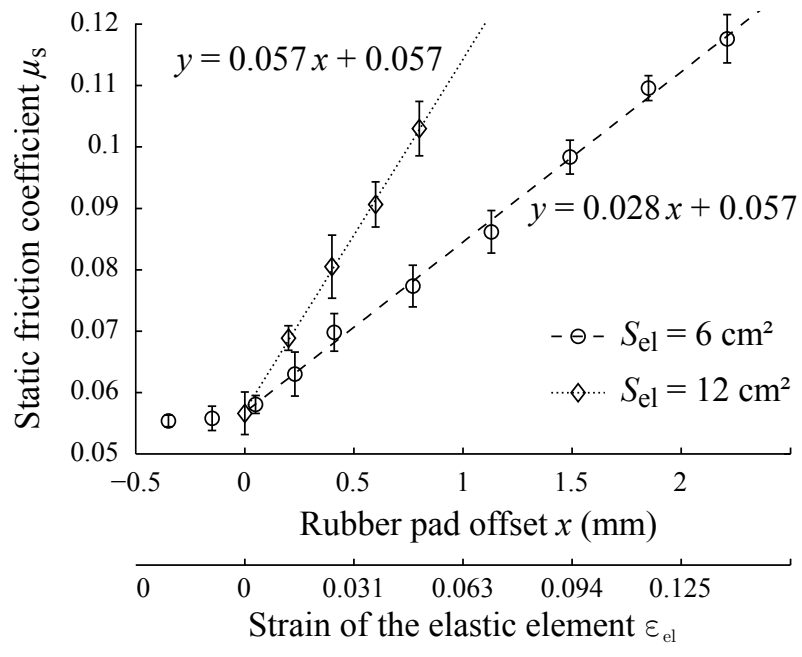

Figure 6: Static friction as a function of offset position for two rubber surfaces of different surface area. The dashed line (for $S_{\mathrm{el}}$ of $6 \mathrm{~cm}^{2}$ ) and dotted line (for $S_{\text {el }}$ of $12 \mathrm{~cm}^{2}$ ) represent linear fits to the data. Considering an uncompressed length of $16 \mathrm{~mm}$, the strain of the elastic elements (foam and rubber) is calculated as $\varepsilon_{\mathrm{el}}=x / 16$.

\subsubsection{Influence of vibration frequency}

Six patterns of sinusoidal vibrations, lasting $5 \mathrm{~s}$, with frequencies ranging from 10 to $35 \mathrm{~Hz}$ were tested. The tile was tilted by $5^{\circ}$, which is close to the static friction angle measured for the PTFE tile $\left(\varphi_{\mathrm{s}} \approx 6.3^{\circ}\right)$. The acceleration amplitude of vibration was the maximum allowed by the controller at the highest frequency tested, which corresponded to a displacement amplitude of $350 \mu \mathrm{m}$ at $35 \mathrm{~Hz}$. The acceleration measured at the center of pressure (CoP) was kept approximately constant at $0.9 \mathrm{~g} \pm 0.1 \mathrm{~g}$ at the beginning of the stimulus, and decreased as the mass slid toward the non-actuated side of the tile, as a result of the applied vibration.

Figure 7 shows the velocity of the CoP in response to different vibration frequencies for one trial, with analysis up to a displacement of $10 \mathrm{~cm}$. A dashed line indicates the average velocity of the corresponding displacement. Sliding speed was approximately constant with applied acceleration ranging from 10 to $25 \mathrm{~Hz}$.

\subsubsection{Influence of vibration amplitude}

The influence of vibration amplitude was investigated informally by adding vibrations ranging from $0.005 \mathrm{~g}$ to $0.7 \mathrm{~g}$ in peak amplitude at $20 \mathrm{~Hz}$. Five inclinations, ranging between $1.5^{\circ}$ and $5^{\circ}$, were tested for 7 different amplitudes. Five repetitions for each combination of angle and amplitude were conducted, with the results of each combination averaged together and shown in Fig. 8. The minimum amplitude of vibration, around $0.01 \mathrm{~g}$, necessary to induce sliding, was consistent over the inclinations tested. The results also suggest that acceleration has a proportional effect on the velocity of the $\mathrm{CoP}$, and therefore, on the apparent coefficient of kinetic friction. Our of $0.05 \mathrm{~g}$ RMS of acceleration results in a reduction of the 


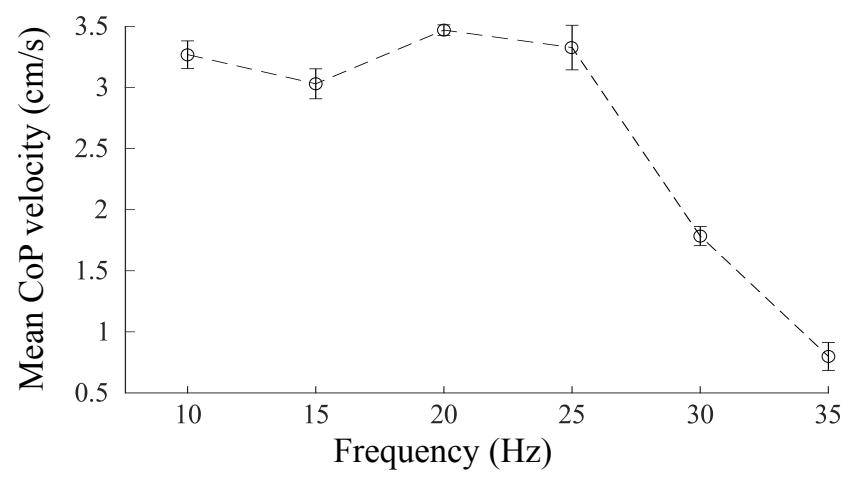

Figure 7: Mean velocities at the center of pressure for frequencies ranging from 10 to $35 \mathrm{~Hz}$ at an angle of inclination of $5^{\circ}$ for five different test sequences. Error bars correspond to a single standard deviation.

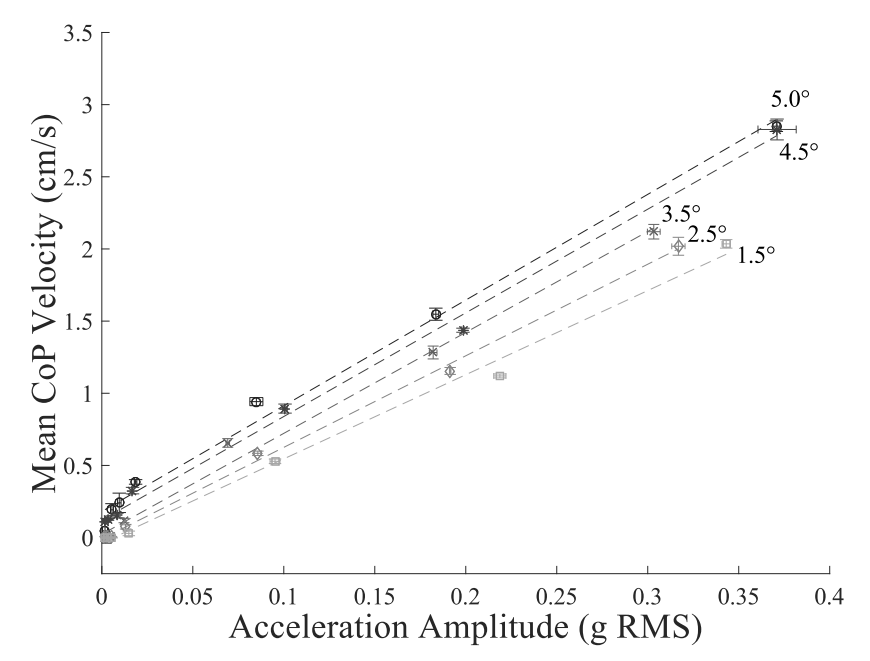

Figure 8: Mean velocities at the center of pressure for various vibration amplitudes and angles of inclination at $20 \mathrm{~Hz}$. The dashed lines represent linear fits to each angle's data. Error bars correspond to a single standard deviation.

coefficient of friction of approximately 0.04 .

Although the results of these experiments demonstrate the feasibility of adding vibration to control the coefficient of friction, this approach suffers from some important limitations, as discussed below in Section 5.2.

\section{Discussion}

Table 1 provides a comparison of the strengths and weaknesses of the various design approaches presented earlier in the paper. This includes those approaches used in earlier slip-related studies, technologies that we consider inadequate for our needs, and the approaches investigated here, that is, the use of rolling elements, vibration, and low-friction surface coverings to achieve a variable coefficient of friction. 
Table 1: Comparison table of design approaches for variable-friction walking devices

\begin{tabular}{|c|c|c|c|c|c|c|}
\hline & Strategies & Minimum $\mu_{\mathrm{s}}$ & Isotropy & $\begin{array}{l}\text { Ease of } \\
\text { control }\end{array}$ & $\begin{array}{c}\text { Distortion of } \\
\text { virtual texture }\end{array}$ & Limitations \\
\hline \multirow{3}{*}{ 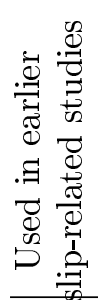 } & $\begin{array}{l}\text { Changing materials or lubrication } \\
{[40,41,42,28,33,32,38,43,34]}\end{array}$ & 0.02 & + & - & + & no friction variation \\
\hline & $\begin{array}{l}\text { Rollers } \\
{[29,47,35]}\end{array}$ & 0.04 & - & + & - & $\begin{array}{l}\text { uneven surface, anisotropy, } \\
\text { vibrotactile noise, visibility }\end{array}$ \\
\hline & $\begin{array}{l}\text { Rollers with mobile platform } \\
{[30,49,27]}\end{array}$ & 0.04 & - & + & - & $\begin{array}{l}\text { inertia, anisotropy, } \\
\text { vibrotactile noise }\end{array}$ \\
\hline \multirow{3}{*}{ 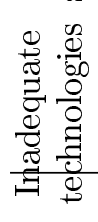 } & Air bearings & 0 & + & - & + & requires constant contact surface \\
\hline & Ultrasonic vibrations & 0 & + & - & + & very limited load (fingertip) \\
\hline & Electromagnetic repulsion & 0 & + & - & + & unsafe, heavy magnet in shoes \\
\hline \multirow{4}{*}{ 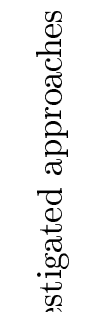 } & Ball transfer units & 0.03 & + & + & - & $\begin{array}{l}\text { vibrotactile noise, } \\
\text { in floor: unevenness, visibility } \\
\text { in shoe: weight }(6 \mathrm{BTUs} \approx 600 \mathrm{~g})\end{array}$ \\
\hline & $\begin{array}{l}\text { Low-friction coverings } \\
\text { with } 20 \mathrm{~Hz} \text { vibrations }\end{array}$ & 0.05 & + & + & - & $\begin{array}{l}\text { requires powerful actuation, } \\
\text { vibrotactile and acoustic noise }\end{array}$ \\
\hline & Low-friction coverings with pins & 0.06 & + & + & + & wears down quickly \\
\hline & Mixed-friction coverings & 0.06 & + & + & + & minimum $\mu_{\mathrm{s}}$ around 0.06 \\
\hline
\end{tabular}




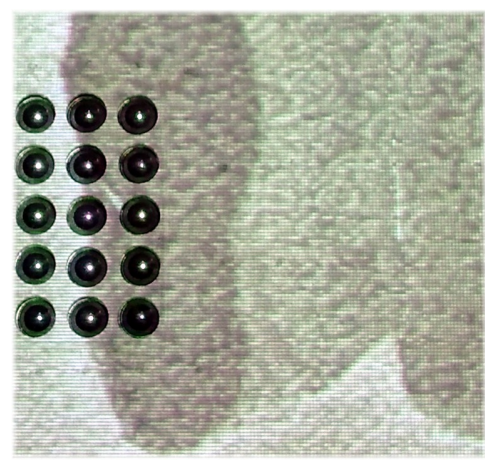

Figure 9: Visual projection of a virtual sandy ground on the BTUbased tile.

\subsection{Limitations of BTUs}

Although the PTFE coverings are not able to induce the same minimum coefficient of friction as the BTU mechanism, they offer some important advantages. Specifically, they provide a flat surface, whereas the BTU balls form $\mathrm{an}^{75}$ uneven surface that does not simulate typical floors. This can have significant perceptual consequences, depending on the thickness and stiffness of the soles. Moreover, the spherical surface of BTUs creates high contact pressure bethe balls and the walker, which deforms the sole of the foot or the shoe, particularly with soft soles. This can result in the sole making contact not only with the large balls of the BTUs but also with the cover plate, which in turn affects the friction between the surfaces in an uncontrollable way.

Another constraint of BTUs is the amount of space be-765 tween the BTUs due to their frame, exacerbating the problem of an uneven surface. This prevents the simulation of realistic heel strike if the foot is highly inclined, such as during long strides. This limitation could be reduced with a denser array of units and a rounded heel edge. Smaller ${ }_{70}$ commercial BTUs, known as miniature BTUs, contain a $\varnothing 5 \mathrm{~mm}$ ball, but their frame is relatively larger compared to that of glide units. To avoid the heel contacting the frame between the balls, the heel would need to be sufficiently rounded.

Finally, visibility of the balls is hard to avoid and would impede a seamless visual display in a virtual environment. This is illustrated in Fig. 9 with the projection of a virtual are reflective and cannot be covered with paint while non $\rightarrow$ so reflecting balls made out of nylon cannot bear sufficient load. Special BTUs with a nonreflecting coating and resistant to high contact pressure could help, but the overall cost would likely be prohibitive.

The vibrotactile display also is limited. The motion of 85 the large ball of the BTUs against the smaller bearings produces auditory and tactile sensations that interfere with the vibrotactile display. Additionally, the sound of any impact against the BTUs is distinctly metallic. These perturbations can significantly affect the recognition of groundso materials [65] and in turn, reduce the realism and the sense of presence of the simulation.

In summary, the use of BTUs for a VFFD raises potential issues with respect to several modalities: tactile (shape and vibrotactile), visual, and auditory. Considering those limitations, we confined our subsequent investigation to the use of low-friction coverings.

\subsection{Limitations of vibration}

Both the frequency and amplitude of vibrations, induced by the actuator, may be used to control the coefficient of friction. However, such vibrations also generate acoustic noise, which could be sufficiently loud to disrupt the immersive sensory experience of a walker. Moreover, the friction reduction measured in our experiment was associated with a corresponding sliding velocity above which the friction reduction is likely to be smaller, as studied in [62]. In a context of shoe-floor contact, this means that the friction reduction will depend on the sliding velocity applied by the walker.

Since the risk of falling is predominant at the point of heel strike, it is possible to reduce friction through added vibrations only during that brief portion of the gait phase. This could be facilitated by an instrumented tile, enabling force sensing, which allows measurement of the first heel strike. Nevertheless, the added noise, even limited to this short period, would be sufficiently disturbing to the quality of the simulation, e.g., of cracking ice or snow.

\subsection{A Next-Generation Shoe Design}

Our analysis of the various options for achieving dynamic friction, as summarized in Table 1, identified various limitations in all the approaches considered. The most promising options with respect to our design objectives were those that employed low- or mixed-friction coverings of the shoe itself. These approaches ensure isotropy of friction, support ease of control, and in the case of projected graphics, avoid distortion of the visual texture. In addition, they offer the important benefit that the mechanism responsible for adjustment of friction need not involve modification of the surrounding environment, e.g., the floor surface. Otherwise, the approaches are only viable within a controlled environment, e.g., a laboratory, where the necessary modifications can be applied and maintained. For general use outside of a laboratory, it is imperative that the variable friction mechanism be confined entirely within the shoe itself.

Following from this analysis, we undertook the design of a novel variable-friction shoe, described here. This design employs low-friction coverings and a higher-friction elastic element whose deformation is adjusted by a mechanism located under the sole.

The implementation of this design beneath a shoe must take into account the different potential locations and orientations of contact. Regardless of contact location, both low- and high-friction surfaces must be present. The main 
controlled

pressing mechanism

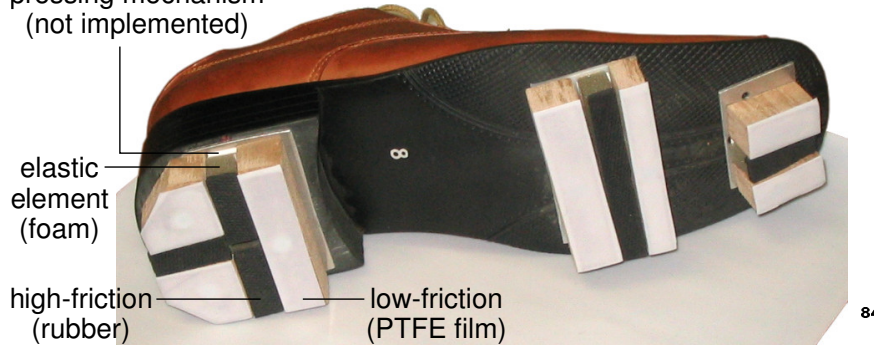

Figure 10: Variable-friction shoe with PTFE and rubber surfaces.

contact events occur on the edges of the shoe, particularly $y_{845}$ under the heel and the toe. Therefore, in our first implementation shown in Fig. 10, several groups of a symmetric PTFE-rubber-PTFE set were positioned such that the friction can be controlled for all essential striking points. The areas of PTFE should be small enough to ensure ${ }_{\mathbf{8 5 0}}$ sufficient contact pressure to achieve low friction, as recommended by Dupont [39]. Minimizing the PTFE area also reduces the accumulation of dust, thereby maintaining performance and reducing the maintenance requirements. Using adhesive PTFE films further facilitates their ${ }_{855}$ replacement when they are worn.

To keep the height of the device small, and reduce potential perceptual bias from a high sole, the control mechnism should be small and flat. However, the mechanism must be sufficiently fast in adjusting the position of the ${ }_{\mathbf{8 6 0}}$ elastic element in order to maintain a constant coefficient of friction during a stride. Although the displacement of the elastic elements is small, the mechanism must be able o bear the weight of a human, ideally without additional energy supply. A suitable mechanism for this purpose could be based on a leadscrew or wedge.

In any case, there is a trade-off between the compactness of the mechanism, its speed of operation, and its ability to $\mathrm{g}_{\mathbf{8 6}}$ 815 compress the elastic elements under the weight of a human. The first half of a stride requires only that the mechanism act quickly. As the number of friction elements of the shoe contacting the floor gradually increases, the elastic elements must be expanded to maintain the target friction ${ }_{\mathbf{8 7}}$ The weight in this first phase facilitates the expansion. The most challenging aspect of such a next-generation shoe is likely to appear during the second half of a stride, that is, during propulsion. In this phase, the elastic element under the toe becomes the only one controlling friction and must ${ }_{\mathbf{8 7}}$ therefore be compressed by the mechanism accordingly to maintain the target friction. In this regard, it may prove difficult to achieve the necessary rapid compression with a compact actuator.

One limitation of the variable-friction shoe using soft rubbing areas and employing elastic elements is that the effective friction is not controlled during the short transient period when the elastic element is compressed at the time of foot strike. The duration of this transient period depends on the velocity of the foot strike, in addition to
335 the stiffness and offset position of the elastic element. Although this period may be negligible, its importance in the risk of slipping asks for it to be investigated.

\section{Conclusions and Future work}

We proposed several approaches to design a variablefriction device for walking interaction, including omnidirectional rolling elements such as BTUs, as well as lowfriction coverings, either embedded in the floor or beneath the shoe. Measurements of static friction showed that a device based on rolling elements can simulate coefficients of friction as low as ice but requires specific footwear and has several limitations with respect to tactile, visual, and auditory modalities.

The embedding of a BTU-based mechanism in the floor requires a dense array of elements to provide sufficient spatial resolution, which implies a costly and non-portable solution. An alternative design, employing a combination of low- and high-friction materials beneath the shoe, is reasonably portable and offers adequate spatial resolution, while satisfying additional requirements related to use in a multimodal virtual environment. Although dynamic control of friction during a full stride requires additional work, our first tests show very promising results.

In future work, an automated mechanism should be added to the shoe device to control the different friction pads in real-time. Measurements of dynamic friction as well as experiments with human subjects will be carried out to validate that method.

\section{Appendix: Measurement of acceleration of the tile}

Normal acceleration of the tile under the shoes could not be measured with a single accelerometer due to the presence of angular acceleration. This is caused both by the application of force from the low-frequency vibration actuators at the side of the tile and the use of suspension elements under the tile, which permit angular vibrations with regard to the base, depending on the position of the feet. As a result, normal acceleration differs according to the position on the tile where it is measured. Therefore, three accelerometers (Analog Devices model ADXL 320 with a $1 \mathrm{kHz}$ bandwidth) were attached under the tile on three different sides.

Assuming that the tile has no intrinsic rotation, the acceleration $a_{\mathrm{z}}$ normal to the tile at position $(x, y)$ can be calculated from the normal accelerations $a_{i z}$ measured by the three accelerometers, by resolving the following linear system of three equations:

$$
a_{\mathrm{z}}+\dot{\omega}_{\mathrm{x}}\left(y-y_{i}\right)-\dot{\omega}_{\mathrm{y}}\left(x-x_{i}\right)=a_{i \mathrm{z}} \quad \text { for } \mathrm{i}=\{1,2,3\},
$$

where $\left(x_{i}, y_{i}\right)$ are the coordinates of accelerometer $i$, and $\dot{\omega}_{\mathrm{y}}$ and $\dot{\omega}_{\mathrm{y}}$ are the angular accelerations of the tile around its 
tangential axes. Assuming that the three accelerometers are not aligned, the solution of the system is

$$
\begin{aligned}
a_{\mathrm{z}} & =\left(a_{1 z}\left(x\left(y_{2}-y_{3}\right)-y\left(x_{2}-x_{3}\right)+x_{2} y_{3}-y_{2} x_{3}\right)\right. \\
& +a_{2 z}\left(x\left(y_{3}-y_{1}\right)-y\left(x_{3}-x_{1}\right)+x_{3} y_{1}-y_{3} x_{1}\right) \\
& \left.+a_{3 z}\left(x\left(y_{1}-y_{2}\right)-y\left(x_{1}-x_{2}\right)+x_{1} y_{2}-y_{1} x_{1}\right)\right) \\
& \left(x_{1}\left(y_{2}-y_{3}\right)+x_{2}\left(-y_{1}+y_{3}\right)+x_{3}\left(y_{1}-y_{2}\right)\right)^{-1} .
\end{aligned}
$$

\section{Acknowledgments}

This work was supported by a grant from the Ministère du Développement Économique, de l'Innovation et de l'Exportation in coordination with the European Com-945 mission Seventh Framework FET-OPEN project NIW: Natural Interactive Walking and by a post-graduate scholarship from the Fonds québécois de la recherche sur la nature et les technologies. The authors also wish to thank ${ }^{250}$ Yon Visell for his foundational contributions to these efforts.

\section{References}

[1] V. Levesque, L. Oram, K. MacLean, A. Cockburn, N. D. Marchuk, D. Johnson, J. E. Colgate, M. A. Peshkin, Enhancing physicality in touch interaction with programmable friction, in: Proc. SIGCHI Conference on Human Factors in Computing Systems (CHI 2011), ACM, New York, NY, USA, 2011, pp. 2481-2490. doi:10.1145/1978942.1979306.

[2] G. Casiez, N. Roussel, R. Vanbelleghem, F. Giraud, Surfpad: riding towards targets on a squeeze film effect, in: Proc. SIGCHI Conference on Human Factors in Computing Systems (CHI 2011), ACM, New York, NY, USA, 2011, pp. 2491-2500. doi: 10.1145/1978942.1979307.

[3] O. Bau, I. Poupyrev, REVEL: Tactile feedback technology for ${ }_{970}$ augmented reality, ACM Trans. Graph. 31 (4) (2012) 89:189:11. doi: 10.1145/2185520.2185585.

[4] R. P. Darken, W. R. Cockayne, D. Carmein, The omnidirectional treadmill: A locomotion device for virtual worlds, in: Proc. 10th Annual ACM Symp. on User Interface Software ${ }_{97}$ and Technology, UIST '97, ACM, New York, NY, USA, 1997, pp. 213-221. doi:10.1145/263407.263550.

[5] H. Iwata, Walking about virtual environments on an infinite floor, in: IEEE Virtual Reality (VR), Houston, TX, USA, 1999, pp. 286-293. doi:10.1109/VR.1999.756964.

[6] A. De Luca, R. Mattone, P. R. Giordano, H. H. Bulthoff, Control design and experimental evaluation of the $2 \mathrm{~d}$ cyberwalk platform, in: IEEE/RSJ Int. Conf. on Intelligent Robots and Systems (IROS), 2009, pp. 5051-5058. doi:10.1109/IROS. 2009.5354610.

[7] J. M. Hollerbach, Y. Xu, R. Christensen, S. C. Jacobsen, Design specifications for the second generation sarcos treadport locomotion interface, in: Haptics Symposium, Proc. ASME Dynamic Systems and Control Division, Vol. DSC-Vol. 69-2, Orlando, FL, USA, 2000, pp. 1293-1298.

[8] M. C. Schwaiger, T. Thummel, H. Ulbrich, A 2d-motion platform: The cybercarpet, in: 2nd Joint EuroHaptics Conf. and Symp. on Haptic Interfaces for Virtual Environment and Teleoperator Systems. World Haptics 2007, Tsukuba, Japan, 2007, pp. 415-420. doi:10.1109/WHC.2007.1.

[9] H. Iwata, H. Yano, F. Nakaizumi, Gait Master: a versatile locomotion interface for uneven virtual terrain, in: IEEE Virtual Reality (VR), Yokohama, Japan, 2001, pp. 131-137. doi:10.1109/VR.2001.913779.
[10] J. Yoon, J. Ryu, A novel locomotion interface with two 6-dof parallel manipulators that allows human walking on various virtual terrains, The International Journal of Robotics Research 25 (7) (2006) 689-708. doi:10.1177/0278364906067373.

[11] Y. Visell, S. Smith, A. Law, R. Rajalingham, J. R. Cooperstock, Contact sensing and interaction techniques for a distributed, multimodal floor display, in: IEEE 3D User Interfaces (3DUI), Waltham, MA, USA, 2010, pp. 75-78. doi:10.1109/3DUI.2010. 5444718 .

[12] J. M. Hausdorff, D. A. Rios, H. K. Edelberg, Gait variability and fall risk in community-living older adults: a 1-year prospective study, Archives of Physical Medicine and Rehabilitation 82 (8) (2001) 1050-1056. doi:10.1053/apmr.2001.24893.

[13] V. Scott, L. Wagar, S. Elliott, Falls \& related injuries among older canadians: fall-related hospitalizations \& intervention initiatives., Tech. rep., Public Health Agency of Canada (2010).

[14] L. W. Chu, I. Chi, A. Y. Chiu, Incidence and predictors of falls in the chinese elderly, Annals, Academy of Medicine, Singapore 34 (1) (2005) 60-72.

[15] H. Luukinen, K. Koski, L. Hiltunen, S.-L. Kivela, Incidence rate of falls in an aged population in northern finland, Journal of Clinical Epidemiology 47 (8) (1994) 843-850. doi:10.1016/ $0895-4356(94) 90187-2$.

[16] R. Grönqvist, J. Abeysekera, G. Gard, S. M. Hsiang, T. B. Leamon, D. J. Newman, K. Gielo-Perczak, T. E. Lockhart, C.-Y. Pai, Human-centred approaches in slipperiness measurement, Ergonomics 44 (13) (2001) 1167-1199. doi:10.1080/ 00140130110085556.

[17] C. Gao, J. Abeysekera, A systems perspective of slip and fall accidents on icy and snowy surfaces., Ergonomics 47 (5) (2004) 573-598. doi:10.1080/00140130410081658718.

[18] B. E. Maki, K. M. Sibley, S. B. Jaglal, M. Bayley, D. Brooks, G. R. Fernie, A. J. Flint, W. Gage, B. A. Liu, W. E. McIlroy, A. Mihailidis, S. D. Perry, M. R. Popovic, J. Pratt, J. L. Zettel, Reducing fall risk by improving balance control: Development, evaluation and knowledge-translation of new approaches, Journal of Safety Research 42 (6) (2011) 473-485. doi: 10.1016/j.jsr.2011.02.002.

[19] B.-A. J. Menelas, M. J.-D. Otis, A serious game for training balance control over different types of soil, in: Serious Games Development and Applications. Proc. 3rd Int. Conf., SGDA 2012, Vol. 7528 of Lecture Notes in Computer Science, Springer Berlin Heidelberg, Bremen, Germany, 2012, pp. 31-42. doi: $10.1007 / 978-3-642-33687-4 \_3$.

[20] S. V. Adamovich, G. G. Fluet, E. Tunik, A. S. Merians, Sensorimotor training in virtual reality: A review, NeuroRehabilitation 25 (1) (2009) 29-44.

[21] A. Mirelman, P. Bonato, J. E. Deutsch, Effects of training with a robot-virtual reality system compared with a robot alone on the gait of individuals after stroke, Stroke 40 (1) (2009) 169-174. doi:10.1161/strokeaha.108.516328.

[22] J.-F. Esculier, J. Vaudrin, P. Beriault, K. Gagnon, L. E. Tremblay, Home-based balance training programme using wii fit with balance board for parkinson's disease: A pilot study, Journal of Rehabilitation Medicine 44 (2) (2012) 144-150. doi: 10.2340/16501977-0922.

[23] A. Grosjean, E. Fabbri, E. Feldheim, T. Snoeck, M. Amand, C. Keuterickx, C. Balestra, On the use of the wii fit in reducing falling risk factors and improving balance for the elderly, Kinésithérapie, la Revue 10 (107) (2010) 41-45.

[24] L. Pareto, J. Broeren, D. Goude, M. Rydmark, Virtual reality, haptics and post-stroke rehabilitation in practical therapy, in: Proc. 7th Int. Conf. on Disability, Virtual Reality and Associated Technologies, Maia, Portugal, 2008, pp. 245-252.

[25] D. L. Jaffe, D. A. Brown, C. D. Pierson-Carey, E. L. Buckley, H. L. Lew, Stepping over obstacles to improve walking in individuals with poststroke hemiplegia, Journal of Rehabilitation Research and Development 41 (3a) (2004) 283-292. doi: $10.1682 / \mathrm{jrrd} .2004 .03 .0283$.

[26] J. Feasel, M. C. Whitton, L. Kassler, F. P. Brooks Jr., M. D. Lewek, The integrated virtual environment rehabilitation tread- 

(2011) 290-297. doi:10.1109/tnsre.2011.2120623.

[27] T. Bhatt, Y.-C. Pai, Generalization of gait adaptation for fal prevention: from moveable platform to slippery floor., Journal of Neurophysiology 101 (2) (2009) 948-957. doi:10.1152/jn. 91004.2008.

[28] R. Cham, M. S. Redfern, Changes in gait when anticipating slippery floors, Gait \& Posture 15 (2) (2002) 159-171. doi: 10.1016/s0966-6362(01)00150-3.

[29] D. S. Marigold, A. E. Patla, Strategies for dynamic stability during locomotion on a slippery surface: Effects of prior experioso ence and knowledge, Journal of Neurophysiology 88 (1) (2002) 339-353. doi:10.1152/jn.00691.2001.

[30] Y.-C. Pai, Induced limb collapse in a sudden slip during termination of sit-to-stand, Journal of Biomechanics 32 (12) (1999) 1377-1382. doi:10.1016/S0021-9290 (99)00126-8.

[31] C. J. Robinson, M. C. Purucker, L. W. Faulkner, Design, control, and characterization of a sliding linear investigative platform for analyzing lower limb stability (slip-falls), IEEE Trans. Rehabil. Eng. 6 (1998) 334-350. doi:10.1109/86.712232.

[32] D. T.-P. Fong, D.-W. Mao, J.-X. Li, Y. Hong, Greater toe griposo and gentler heel strike are the strategies to adapt to slippery surface, Journal of Biomechanics 41 (4) (2008) 838-844. doi: 10.1016/j.jbiomech.2007.11.001.

[33] K. Beschorner, R. Cham, Impact of joint torques on heel acceleration at heel contact, a contributor to slips and falls, Ergonomicæo95 51 (12) (2008) 1799-1813. doi:10.1080/00140130802136479.

[34] G. Cappellini, Y. P. Ivanenko, N. Dominici, R. E. Poppele, F. Lacquaniti, Motor patterns during walking on a slippery walkway, Journal of Neurophysiology 103 (2) (2010) 746-760. doi:10.1152/jn.00499.2009.

[35] A. R. Oates, J. S. Frank, A. E. Patla, Control of dynamic stability during adaptation to gait termination on a slippery surface, Experimental Brain Research 201 (1) (2010) 47-57. doi: 10.1007/s00221-009-2011-2.

[36] A. D. Roberts, J. C. Richardson, Interface study of rubber-icmos friction, Wear 67 (1) (1981) 55-69. doi:10.1016/0043-1648(81) 90075-2.

[37] A. Klein-Paste, N. K. Sinha, Comparison between rubber-ice and sand-ice friction and the effect of loose snow contamination, Tribology International 43 (5-6) (2010) 1145-1150. doir110 10.1016/j.triboint.2009.12.037

[38] D. T.-P. Fong, Y. Hong, J.-X. Li, Human walks carefully when the ground dynamic coefficient of friction drops below 0.41 , Safety Science 47 (10) (2009) 1429-1433. doi:10.1016/j.ssci. 2009.04.005.

[39] DuPont Fluoroproducts, Teflon ${ }^{\circledR}$ PTFE properties handbook, Tech. Rep. H-37051-3, DuPont ${ }^{T M}$ (1996).

[40] M. G. A. Llewellyn, V. R. Nevola, Strategies for walking on low-friction surfaces, in: Proc. 5th Int. Conf. on Environmental Ergonomics, Maastricht, The Netherlands, 1992, pp. 156-157.1120

[41] A. E. Fendley, M. I. Marpet, Required coefficient of friction versus walking speed: Potential influences of footwear and walkway surfaces, Journal of Testing and Evaluation 24 (6) (1996) 359367. doi:10.1520/jte11458j.

[42] J. P. Hanson, M. S. Redfern, M. Mazumdar, Predicting slipsi25 and falls considering required and available friction, Ergonomics 42 (12) (1999) 1619-1633. doi:10.1080/001401399184712.

[43] J. C. Menant, J. R. Steele, H. B. Menz, B. J. Munro, S. R. Lord, Effects of walking surfaces and footwear on temporo-spatial gaitparameters in young and older people, Gait \& Posture 29 (3i) 30 (2009) 392-397. doi:10.1016/j.gaitpost.2008.10.057.

[44] C. Moore Strobel, P. L. Menezes, M. R. Lovell, K. E. Beschorner, Analysis of the contribution of adhesion and hysteresis to shoe-floor lubricated friction in the boundary lubrication regime, Tribology Letters 47 (3) (2012) 341-347. doir135 10.1007/s11249-012-9989-5

[45] C. T. Moore, P. L. Menezes, M. R. Lovell, K. E. Beschorner, Analysis of shoe friction during sliding against floor material: role of fluid contaminant, Journal of Tribology 134 (4) (2012) 041104. doi:10.1115/1.4007346
[46] S. R. M. Moghaddam, M. S. Redfern, K. E. Beschorner, A microscopic finite element model of shoe-floor hysteresis and adhesion friction, Tribology Letters 59 (3) (2015) 42 . doi: $10.1007 / \mathrm{s} 11249-015-0570-\mathrm{x}$.

[47] A. R. Oates, A. E. Patla, J. S. Frank, M. A. Greig, Control of dynamic stability during gait termination on a slippery surface, Journal of Neurophysiology 93 (1) (2005) 64-70. doi:10.1152/ jn.00423.2004.

[48] G. Millet, M. Otis, G. Chaw, J. R. Cooperstock, Initial development of a variable-friction floor surface, in: Canadian Medical and Biological Engineering Conf. (CMBEC'34), Festival of International Conferences on Caregiving, Disability, Aging and Technology, Toronto, ON, Canada, 2011, pp. 1-4.

[49] F. Yang, Y.-C. Pai, Correction of the inertial effect resulting from a plate moving under low-friction conditions, Journal of Biomechanics 40 (12) (2007) 2723-2730. doi:10.1016/j. jbiomech.2006.12.008.

[50] T. Watanabe, S. Fukui, A method for controlling tactile sensation of surface roughness using ultrasonic vibration, in: Proc. 1995 IEEE Int. Conf. on Robotics and Automation, Vol. 1, 1995, pp. 1134-1139. doi:10.1109/ROBOT. 1995.525433.

[51] L. Winfield, J. Glassmire, J. E. Colgate, M. Peshkin, T-pad: Tactile pattern display through variable friction reduction, in: 2nd Joint EuroHaptics Conf. and Symp. on Haptic Interfaces for Virtual Environment and Teleoperator Systems. World Haptics 2007, Tsukuba, Japan, 2007, pp. 421-426. doi:10.1109/WHC. 2007.105.

[52] M. Biet, F. Giraud, B. Lemaire-Semail, Squeeze film effect for the design of an ultrasonic tactile plate, IEEE Transactions on Ultrasonics, Ferroelectrics and Frequency Control 54 (12) (2007) 2678-2688. doi:10.1109/tuffc.2007.596.

[53] F. Giraud, M. Amberg, B. Lemaire-Semail, G. casiez, Design of a transparent tactile stimulator, in: IEEE Haptics Symp., Institute of Electrical and Electronics Engineers (IEEE), 2012, pp. 485-489. doi:10.1109/haptic.2012.6183835.

[54] M. Wiertlewski, D. Leonardis, D. J. Meyer, M. A. Peshkin, J. E. Colgate, A high-fidelity surface-haptic device for texture rendering on bare finger, in: M. Auvray, C. Duriez (Eds.), Haptics: Neuroscience, Devices, Modeling, and Applications. Proc. 9th Int. Conf., EuroHaptics 2014, Vol. 8619 of Lecture Notes in Computer Science, Springer Berlin Heidelberg, Versailles, France, 2014, pp. 241-248. doi:10.1007/978-3-662-44196-1_ 30.

[55] E. Vezzoli, T. Sednaoui, M. Amberg, F. Giraud, B. LemaireSemail, Texture rendering strategies with a high fidelity - capacitive visual-haptic friction control device, in: F. Bello, H. Kajimoto, Y. Visell (Eds.), Haptics: Perception, Devices, Control, and Applications. Proc. 10th Int. Conf., EuroHaptics 2016, Vol. 9774 of Lecture Notes in Computer Science, Springer International Publishing, London, UK, 2016, pp. 251-260. doi: 10.1007/978-3-319-42321-0_23.

[56] P. Berkelman, M. Dzadovsky, Magnet levitation and trajectory following motion control using a planar array of cylindrical coils, in: ASME Dynamic Systems and Control Conf., Ann Arbor, MI, 2008, pp. 923-930. doi:10.1115/dscc2008-2229.

[57] P. Berkelman, M. Dzadovsky, Novel design, characterization, and control method for large motion range magnetic levitation, IEEE Trans. Magn. 1 (2010) 0500104. doi:10.1109/1mag. 2009. 2039341

[58] C. H. Lewia, Reversible heel, U.S. Patent 6662475 (Dec. 2003).

[59] S. Teteriatnikov, Spinning shoe, U.S. Patent 8104193 (Jan. 2012).

[60] W. Lenkiewicz, The sliding friction process-effect of external vibrations, Wear 13 (2) (1969) 99-108. doi:10.1016/ 0043-1648 (69) 90505- 5.

[61] M. A. Chowdhury, M. Helali, The effect of amplitude of vibration on the coefficient of friction for different materials, Tribology International 41 (4) (2008) 307-314. doi:10.1016/j. triboint.2007.08.005.

[62] E. Teidelt, J. Starcevic, V. L. Popov, Influence of ultrasonic oscillation on static and sliding friction, Tribology Letters 48 (1) 
(2012) 51-62. doi:10.1007/s11249-012-9937-4.

[63] R. Capozza, A. Vanossi, A. Vezzani, S. Zapperi, Triggering frictional slip by mechanical vibrations, Tribology Letters 48 (1) (2012) 95-102. doi:10.1007/s11249-012-0002-0.

1145 [64] Y. Visell, J. R. Cooperstock, Design of a vibrotactile display via a rigid surface, in: IEEE Haptics Symp., Waltham, MA, USA, 2010, pp. 133-140. doi:10.1109/HAPTIC.2010.5444664.

[65] B. L. Giordano, S. McAdams, Y. Visell, J. R. Cooperstock, H. Yao, V. Hayward, Non-visual identification of walking grounds, Journal of the Acoustical Society of America 123 (5) (2008) 3412. doi:10.1121/1.2934136. 